\title{
Elastography improves accuracy of early hepato-biliary complications diagnosis after allogeneic stem cell transplantation
}

Haematologica 2021

Volume 106(9):2374-2383

\section{Correspondence:}

DAVID MICHONNEAU

david.michonneau@aphp.fr

Received: March 5, 2020.

Accepted: July 27, 2020.

Pre-published: July 30, 2020.

https://doi.org/10.3324/haematol.2019.245407

(C)2021 Ferrata Storti Foundation

Material published in Haematologica is covered by copyright. All rights are reserved to the Ferrata Storti Foundation. Use of published material is allowed under the following terms and conditions:

https://creativecommons.org/licenses/by-nc/4.0/legalcode. Copies of published material are allowed for personal or internal use. Sharing published material for non-commercial purposes is subject to the following conditions:

https://creativecommons.org/licenses/by-nc/4.0/legalcode, sect. 3. Reproducing and sharing published material for commercial purposes is not allowed without permission in writing from the publisher. Anne-Marie Zagdanski, ${ }^{2}$ Morgane De Boutiny, ${ }^{2}$ Simona Pagliuca, ${ }^{1}$ Aurélien Sutra de Galy, ${ }^{1}$ Marie Robin,${ }^{1}$ Régis Peffault de Latour, ${ }^{1,4}$ Aurélie Plessier, ${ }^{3}$ Flore Sicre de Fontbrune, ${ }^{1}$ Aliénor Xhaard, ${ }^{1}$ Pedro Henrique Prata, ${ }^{1}$ Dominique Valla, ${ }^{3,4}$ Gérard Socie ${ }^{1,4 \#}$ and David Michonneau ${ }^{1,4 \#}$

${ }^{1}$ Hematology and Transplantation Unit, Saint Louis Hospital, APHP, Paris; ${ }^{2}$ Radiology Unit, Saint Louis Hospital, APHP, Paris; ${ }^{3}$ DHU Unit, Pôle des Maladies de l'Appareil Digestif, Service d'Hépatologie, Centre de Référence des Maladies Vasculaires du Foie, Hôpital Beaujon, AP-HP, Clichy and ${ }^{4}$ Université de Paris, INSERM U976, Paris, France

*PED and PB contributed equally as co-first authors. "GS and DM contributed equally as co-senior authors

\section{ABSTRACT}

ignificant morbidity and mortality have been associated with liver complications after allogeneic hematopoietic stem cell transplantation (allo-HSCT). Causes and consequences of these hepato-biliary complications are various and might be life-threatening. A high misdiagnosis rate has been reported because of a weak correlation between clinical, laboratory and imaging data. Liver elastography, a liver stiffness measure, is able to assess liver fibrosis and portal hypertension in most liver diseases, but data after allo-HSCT are scarce. Our aim was to determine the interest of sequential liver stiffness measurements for the diagnosis of early hepatic complications after allo-HSCT. Over a 2-year time period, 161 consecutive adult patients were included and 146 were analyzed. Ultrasonography and elastography measurements were performed before transplantation, at day+7 and day +14 by three different experienced radiologists unaware of the patients' clinical status. Eightyone $(55 \%)$ patients had liver involvements within the first 100 days after allo-HSCT. Baseline elastography was not predictive for the occurrence of overall liver abnormalities. A significant increase in two-dimensional real-time shearwave elastography (2D-SWE) was found in patients with sinusoidal obstruction syndrome (SOS). Fifteen patients $(10 \%)$ fulfilled European Society for Blood and Marrow Transplantation (EBMT) score criteria and twelve (8\%) reached Baltimore criteria for SOS diagnosis, but only six $(4 \%)$ had a confirmed SOS. 2D-SWE at day+14 allowed early detection of SOS (AUROC $=0.84, P=0.004$ ) and improved sensibility (75\%), specificity (99\%) and positive predictive value $(60 \%)$ over the Seattle, Baltimore or EBMT scores. A 2D-SWE measurement above $8.1 \mathrm{kPa}$ at day+14 after allo-HSCT seems a promising, non-invasive, and reproducible tool for early and accurate diagnosis of $\mathrm{SOS}$.

\section{Introduction}

Over the past two decades, overall survival rate after allo-HSCT has improved. ${ }^{1}$ However, transplantation-related mortality (TRM) remains a significant cause of death, with a reported $15 \%$ to $35 \%$ rate in the current era. ${ }^{1}$ Hepato-biliary complications lead to significant morbidity and TRM after allo-HSCT. ${ }^{2}$ They include liver graft-versus-host disease (GvHD), sinusoidal obstruction syndrome (SOS), drug-induced hepatotoxicity, cholangitis lenta, malignant infiltration, iron overload, hemodynamic modification and biliary obstruction. ${ }^{2}$ Previous studies have reported a higher TRM rate in patients who had a high level of bilirubinemia 
(above $4 \mathrm{mg} / \mathrm{dL}$ or $68 \mu \mathrm{mol} / \mathrm{L}$ ) after allo-HSCT. ${ }^{3}$ More recently, even after reduced-intensity conditioning (RIC), $20 \%$ to $26 \%$ of patients were reported to have a bilirubin peak above $4 \mathrm{mg} / \mathrm{dL}$, which was also associated with poor outcomes. ${ }^{1,4}$ Clinical, biological and imaging data are weekly correlated and can lead to delayed or wrong diagnoses..$^{5}$ Although liver biopsy is useful to establish diagnosis, it has limitations including its feasibility in severely-ill patients and bleeding risk in case of severe thrombocytopenia. ${ }^{5}$ Several clinical scores have been developed to diagnose SOS after allo-HSCT ${ }^{6,7}$ but currently lack of specificity and sensibility. ${ }^{8}$ We previously observed that among patients who fulfilled clinical criteria for SOS diagnosis, less than a half had a final diagnosis of SOS after liver biopsy. ${ }^{5}$ Pitfall associated with ultrasonography and Doppler are due to heterogeneity and lack of reproducibility ${ }^{8,9}$ and to the late onset of some radiological signs (e.g., reverse flow in the portal vein) that can occur in SOS. There are currently no early ultrasonography specific signs that are able to discriminate these various hepatic complications. ${ }^{8}$ Liver stiffness measurement using elastography is widely used and recommended for the assessment of liver fibrosis, cirrhosis and portal hypertension. ${ }^{11,12}$ Different techniques have been described: transient elastography (TE with FibroScan ${ }^{\circledR}$ ), point shear wave with acoustic radiation force impulse (ARFI), and two-dimensional real-time shear wave (2D-SWE). ${ }^{1,13}$ The objective of this study was to determine the feasibility and interest of sequential measures of liver elastography for the diagnosis of early hepatic complications after allo-HSCT.

\section{Methods}

\section{Patients}

Between July 2017 and July 2019, 212 patients underwent an allo-HSCT in the Saint Louis Hospital (Paris, France). A total of 161 patients were included. This study has been conducted in compliance with the Declaration of Helsinki. All patients gave their written consent for the registration of clinical and biological data (CNIL number 2093819), were collected and processed anonymously in a dedicated study (CNIL number 2211540), with authorization of the IRB 00003888 (study number 20-697).

\section{Ultrasonography and elastography}

Ultrasonography, Doppler, and elastography were performed at baseline, at day+7, and at day+14. Two methods were used for elastography for all patients: transient elastography with Fibroscan ${ }^{\circledR}$ (Echosens, Paris, France) and 2D-SWE (Aixplorer, SuperSonic Imaging SA, Aix-en-Provence, France) with a $3.5 \mathrm{MHz}$ convex ultrasound probe (SCX-6-1) and a 7.5 Mhz linear ultrasound probe (SL-10-2). For all ultrasonography and Doppler examination, the following criteria, were assessed: liver and splenic measurements, measurement of the gallbladder wall, ascites, portal vein diameter, portal vein direction flow and maximal flow velocity, spectral waveforms of the hepatic veins. Based on Lassau et al., ${ }^{6}$ and European Society for Blood and Marrow Transplantation (EBMT) classification, an ultrasound-Doppler score based on seven criteria was performed: (i) hepatomegaly, (ii) splenomegaly, (iii) gall bladder wall thickening, (iv) dilatation of main portal vein, (v) ascites, (vi) decrease mean velocity of portal vein, (vii) hepatofugal flow or no flow in portal vein. An additional ultrasonography and elastography could be performed at the discretion of the physician.

\section{Liver test and definition of liver involvements}

Liver involvement was considered if increased serum aspartate aminotransferase (AST) or alanine aminotransferase (ALT) level above twice the upper limit of normal values or hyperbilirubinemia (above $17 \mu \mathrm{mol} / \mathrm{L}$ ) occurred in two consecutive measures. All medical records were retrospectively reviewed to determine the final liver diagnosis. GvHD was graded according to the modified Glucksberg's classification. ${ }^{17}$ When patients had no other organ involvement than suspected liver GvHD, a biopsy was performed to ascertain the diagnosis $(n=3)$. SOS diagnosis was suspected when EBMT, Baltimore or modified Seattle clinical criteria were present in patients. ${ }^{18-20}$ Diagnosis was retained only if proven on liver biopsy $(n=3)$, or using ultrasonography and Doppler criteria, as described in EBMT classification. ${ }^{20}$ If not proven on biopsy or ultrasonography, SOS diagnosis was considered only in the absence of infectious disease, drug toxicity or $\mathrm{GvHD}(\mathrm{n}=3)$, as recommended by the European Association for the Study of the Liver (EASL) guidelines. ${ }^{8,21}$ Drug-induced liver injury (DILI) was defined according to EASL guidelines. ${ }^{22}$

\section{Statistical analysis}

Two-group comparisons were performed with Mann-Whitney $\mathrm{U}$ test and multiple comparisons were performed with KruskalWallis test followed by a Dunn's correction for multiple comparisons. Two-way ANOVA test followed by Dunnet correction was used for multiple comparisons of data with normal distribution and equal variance. ROC curves were built for continuous variable and area under the ROC curve (AUROC) was calculated for SOS diagnosis using all ultrasound and Doppler criteria, 2D-SWE and TE measurements at baseline, day+7 and day+14. Best cutoff value was determined using Youden index. Scores performance were calculated using an intention to diagnose approach using $3 \times 2$ table, as previously described to assess performance of diagnostic tests. ${ }^{21,23}$ All statistical tests were two-tailed with a significance level of 0.05 .

\section{Results}

\section{Population overview}

Over two years, 146 patients out of 161 consecutive patients were analyzed (Figure 1). Fifteen patients were excluded for analysis due to incomplete ultrasonography evaluations. Main patient, disease and transplant features are summarized in Table 1 and Table 2. Median follow-up was 9.2 months (range, 3-19). Five donors had prior hepatitis $B$ virus (HBV) hepatitis, but none had viral replication at the time of stem cell collection. None of the patients had detectable HBV DNA or hepatitis C virus (HCV) RNA. Two patients had Child-Pugh A cirrhosis (previous HBV infection and telomeropathy, one each). Eighteen $(12 \%)$ and seven $(5 \%)$ patients had prior cholecystectomy or splenectomy, respectively and could not be evaluated for all the ultrasonography criteria. During follow-up, 32 patients $(22 \%)$ died, including $16(11 \%)$ early deaths (before day+100). The leading cause of early death was TRM (93\%), including three patients with SOS and two with liver GvHD who had all received a RIC regimen.

\section{Incidence of hepatic involvements after allogeneic hematopoietic stem cell transplantation}

Eighty-one (55\%) patients had a hepatic involvement defined by an elevation of liver enzymes and/or hyperbilirubinemia during the first 100 days after allo-HSCT (Figure 2). Hepatic GvHD was diagnosed in 11 patients 
(including three cases that were proven after liver biopsy) and were associated with skin or gut GvHD in all cases but one. Liver tests improved after GvHD treatment in six cases. SOS diagnosis was suspected when patients had clinical criteria based on classifications (EBMT, Seattle and Baltimore). SOS diagnosis was retrospectively retained when proven on liver biopsy $(n=3)$ or in patients with usual ultrasonography and Doppler criteria $(n=3)$, in the absence of other causes (GvHD, infectious or drugrelated) and according to the clinical evolution. Druginduced liver injury was observed in 48 patients after exposure to drugs within the conditioning regimen $(n=32)$, azole antifungal therapy $(n=12)$ or cyclosporine $A$ $(n=4)$, with normal imaging, no clinical sign for SOS or $\mathrm{GvHD}$, and no positive biological test for infectious disease. All improved after drug withdrawal. A liver biopsy was performed in ten $(7 \%)$ patients when liver blood test abnormalities were not explained by clinical, laboratory or imaging results. Diagnosis was established after pathology analysis in nine patients: SOS $(n=3)$, GvHD $(n=3)$, DILI ( $n=1)$, HEV infection $(n=1)$ and cholangitis lenta $(n=1)$. One biopsy was not conclusive. Of the eight patients in whom liver biopsy was performed using the transjugular route, six had a hepatic venous pressure gradient (HVPG) $<10 \mathrm{mmHg}$ and did not have a SOS, while the two patients with an HVPG $>10 \mathrm{mmHg}$ had a confirmed SOS in biopsy.

\section{Elastography baseline values are not associated with the occurrence of liver involvements after allogeneic hematopoietic stem cell transplantation}

Median basal values before transplantation were $5.4 \mathrm{kPa}$ (interquartile range [IOR], 4.1-6.8) for transient elastography and $6.0 \mathrm{kPa}$ (IOR, 4.9-7.8) for 2D-SWE (Figure 3A). Mean elastography measures from the three radiologists were not significantly different, suggesting that they were not dependent of the operator $(P=0.39$; Online Supplementary Figure S1). TE measure was obtained in 413 out of 432 procedures (two failures at baseline, five at day+7 and 12 at day+14). TE measures did not reach qual-

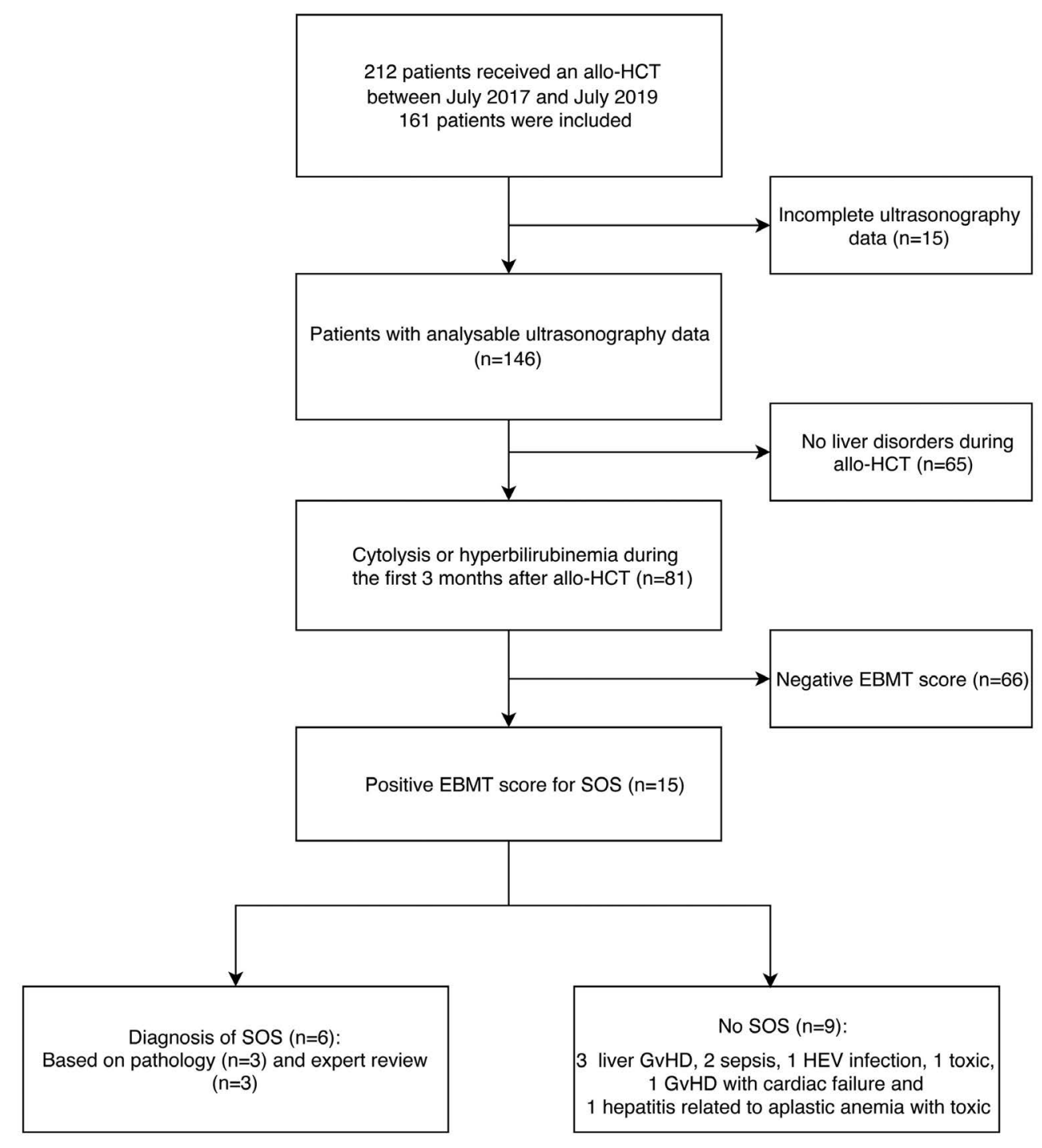

Figure 1. Patients flowchart. Out of 212 patients, 161 patients consented to the study and were transplanted between July 2017 and July 2019 , 146 had a baseline evaluation followed by sequential ultrasonography and elastography measures at day 7 and day+14. Eighty-one patients developed liver blood tests disorders, among which 15 fulfilled European Society for Blood and Marrow Transplantation (EBMT) criteria for sinusoidal obstruction syndrome (SOS) diagnosis, including six with a proven SOS. 
Table 1. Patients' characteristics before transplant.

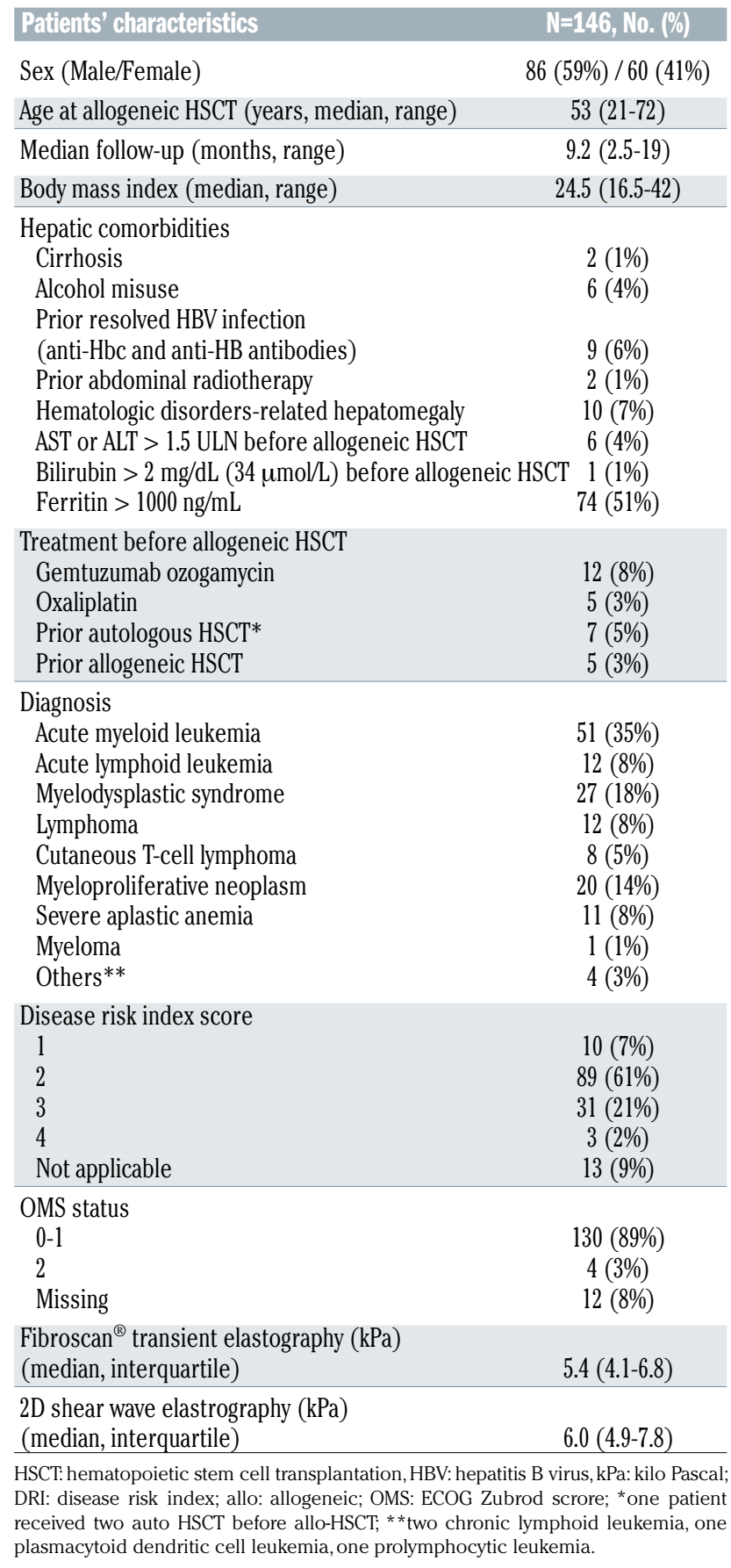

ity criteria (IOR/M <0.3) from international guidelines ${ }^{16}$ and were excluded from analysis in $10.8 \%$ of procedures $(n=45)$. A 2D-SWE measure was obtained in 414 procedures out of 432 (seven failures at baseline, six at day+7 and five at day+14). Overweight (body mass index [BMI] $\left.>29 \mathrm{~kg} / \mathrm{m}^{2}\right)$ was associated with 2D-SWE failure $(P=0.004)$ but not with TE failure $(P=0.21)$. Ascites did not influence the risk of $2 \mathrm{D}-\mathrm{SWE}(P=0.1)$ or TE $(P=0.09)$ failure. Patients with history of liver abnormalities before transplantation had significantly higher baseline values than other patients for TE $(7.9 \mathrm{kPa}$ vs. $5.25 \mathrm{kPa}$, respectively, $P=0.0003$ ) and 2D-SWE $(9 \mathrm{kPa}$ vs. $5.7 \mathrm{kPa}$, respectively, $P=0.004)$. However, the mean baseline value of elastography was not significantly different between patients who
Table 2. Allogeneic hematopoietic stem cell transplantation characteristics.

\begin{tabular}{lc} 
Transplantation characteristics & $N=146$, No. $(\%)$ \\
Donor & \\
HLA-matched-related & $39(27 \%)$ \\
HLA-matched-unrelated & $77(53 \%)$ \\
HLA-mismatched unrelated & $5(3 \%)$ \\
Haplo-identical & $18(12 \%)$ \\
Umbilical cord blood & $1(1 \%)$ \\
Source & \\
Peripheral blood stem cells & $132(90 \%)$ \\
Bone marrow & $13(9 \%)$ \\
Umbilical cord blood & $1(1 \%)$ \\
\hline Donor & \\
Age (median, range) & $30(18-67)$ \\
Male/Female & $91(62 \%) / 55(38 \%)$ \\
CMV seropositivity & $70(48 \%)$ \\
Conditioning & \\
Myeloablative & $28(19 \%)$ \\
Sequential & $6(4 \%)$ \\
Reduced intensity & $100(68 \%)$ \\
Non myeloablative & $12(8 \%)$ \\
TBI-based conditioning & $16(11 \%)$ \\
Anti-thymocyte globulin & $95(65 \%)$ \\
GvHD prophylaxis & \\
CSA+MTX & $37(25 \%)$ \\
CSA+MMF & $82(56 \%)$ \\
CSA+MMF+Cy & $20(14 \%)$ \\
CSA & $6(4 \%)$ \\
Other & $1(1 \%)$ \\
GvHD grading & \\
Grade II-IV & $61(42 \%)$ \\
Grade III-IV & $20(14 \%)$ \\
\hline & \\
\hline &
\end{tabular}

TBI: total body irradiation; GvHD: graft-versus-host disease; CSA: cyclosporin, MTX methotrexate, MMF: mycophenolate mofetil; Cy: post transplantation cyclophosphamide; CMV: cytomegalovirus.

developed liver involvements after transplantation and those who did not, for both TE $(6.4 \mathrm{kPa} v s .6 .0 \mathrm{kPa}$, respectively, $P=0.71)$ and $2 \mathrm{D}-\mathrm{SWE}(7.0 \mathrm{kPa} v s .6 .8 \mathrm{kPa}$, respectively, $P=0.71$ ) (Figure $3 \mathrm{~B}$ ).

\section{Sinusoidal obstruction syndrome diagnosis is associated with an increase in two-dimensional real-time shear wave elastography}

In order to determine if repeated measures of elastography could improve or precede clinical diagnosis of liver disease, additional measures of TE and 2D-SWE were systematically performed at day+7 and day+14 after alloHSCT. Eight (5\%) patients were not evaluable at all time points. Three patients had missing exam at day +7 related to intensice care unit (ICU) transfer $(n=1)$ for acute renal failure (without hepatic disorder) or to missing imaging (without hepatic disorder) $(n=2)$. Five patients had missing examination at day+14 related to ICU transfer $(n=3)$ with early death before day+14 (one SOS, one GvHD/congestive heart failure and one with invasive fungal infection) or because of missing data $(n=2)$ for patients with hepatic GvHD (one confirmed by hepatic biopsy and the others with gut GvHD confirmed with pathologic gut biopsy and improvement after immunosuppressive treatment). Liver stiffness value measured using TE or 2D-SWE was neither different at day+7 nor at day+14 between patients developing or not hepatic complications after allo-HSCT (Figure $3 \mathrm{C}$ and D). Similarly, change in liver stiffness value 
A

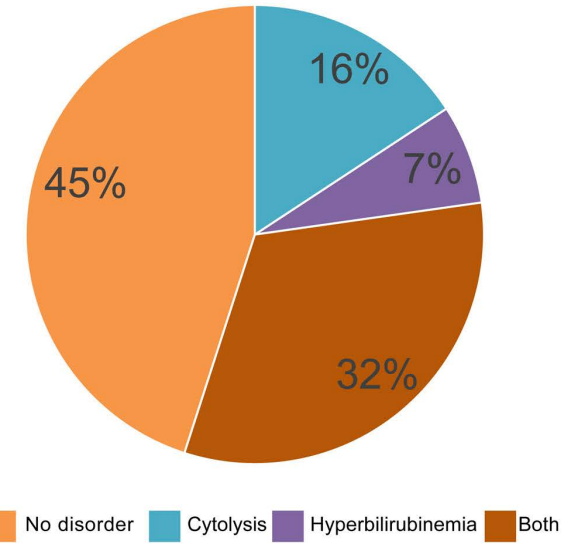

B

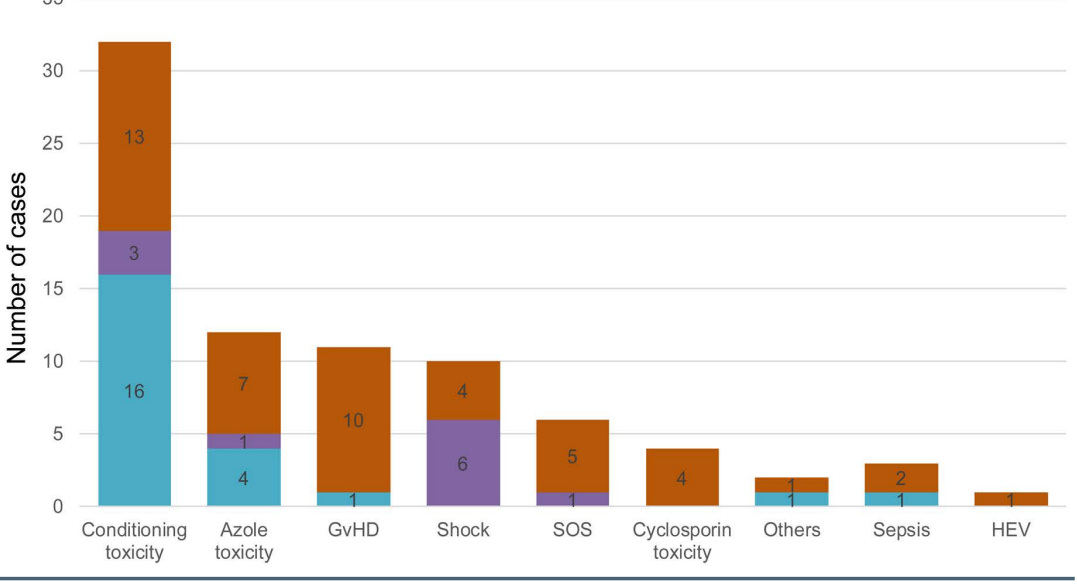

Figure 2. Description of liver blood tests involvement during the first 100 days after allogeneic hematopoietic stem cell transplantation. (A) Pie chart representing frequency of hepatic biological involvements (cytolysis, hyperbilirubinemia, or both). (B) Distribution of diagnoses according to hepatic biological disorders (cytolysis, hyperbilirubinemia, or both). Others are malignant infiltration $(n=1)$ and aplastic anemia-related autoimmune hepatitis ( $n=1)$.

measured using TE or 2D-SWE between baseline and day +7 or day+14 was not associated with hepatic complications after allo-HSCT. We then compared the liver stiffness change from a baseline value to day +7 or day +14 according to each type of liver disease and observed a significant increase only in patients who developed SOS using 2D-SWE at day+14 $(+4.15 \mathrm{kPa} v s .-0.5 \mathrm{kPa}$ in patients without liver involvement, $P=0.02$ ) (Figure $3 \mathrm{E}$ and $\mathrm{F}$ ). We also observed a significant increase in patients who developed SOS versus other liver complications using 2D-SWE at day $+14(+4.15 \mathrm{kPa} v s$. $-0.57 \mathrm{kPa}, P=0.018)$.

\section{Two-dimensional real-time shear wave elastography improves positive predictive value of sinusoidal obstruction syndrome diagnosis scores}

Fifteen patients $(10 \%)$ fulfilled EBMT criteria for SOS diagnosis, ${ }^{20}$ but only six $(4 \%)$ had a confirmed SOS diagnosis after retrospective review of medical history (as described in the Methods section) including two lateonset SOS (Online Supplementary Table S1). The other diagnoses were liver $\operatorname{GvHD}(n=3)$, sepsis $(n=2)$, cyclosporine cholestasis $(n=1)$, HEV infection ( $n=1)$, liver GvHD with congestive heart failure $(\mathrm{n}=1)$, and aplastic anemia-related hepatitis with drug induced liver injury $(\mathrm{n}=1)$. According to the Seattle score, twenty-seven (18\%) patients had criteria for SOS diagnosis ${ }^{24}$ which was confirmed in only five. Twelve patients (8\%) fulfilled SOS criteria according to the Baltimore score, ${ }^{19}$ including four confirmed SOS (Figure 4A and B). Median time between allo-HSCT and SOS diagnosis was 14 days (range, 6-22). Two (6\%) and four $(3.5 \%)$ patients with SOS received myeloablative conditioning (MAC) and RIC regimens, respectively. Three of the four SOS patients with RIC regimen ultimately died. Patients were followed until day+100 after allo-HSCT and no other late-onset SOS was detected. Eight patients, including the two late-onset SOS, had a supplementary ultrasound-Doppler. The higher ultrasonography-Doppler score in patient with SOS diagnosis were $2(n=3)$, and $3(n=3$, including the 2 late SOS), respectively. The two late-onset SOS patients had an increase of TE value at $36 \mathrm{kPa}$ (vs. $12.4 \mathrm{kPa}$ at baseline) and $72 \mathrm{kPa}$ (vs. $5.4 \mathrm{kPa}$ at baseline). No 2D-SWE was available for these two patients. Patients without SOS $(n=6)$ had a median 2D-SWE value at $4.3 \mathrm{kPa}$ and a median TE value at $6.15 \mathrm{kPa}$. Among patients with EBMT criteria, most patients with a SOS diagnosis presented an increase of TE and 2D-SWE measures at day+7 or day+14. In four patients, increased 2D-SWE was recorded at a median of 6 days (range, 1-8) before clinical signs of SOS. 2D-SWE increased 1 day after SOS diagnosis in one patient. Among all ultrasonography, Doppler and elastography measures performed at day+7 or day+14, 2D-SWE value at day+14 was the best marker for SOS diagnosis, with a best cutoff value estimated with Youden index at $8.1 \mathrm{kPa}(P=0.004)$ and a concordance index calculated at 0.84 (AUROC $=0.84$ [0.69-0.95]) (Figure 4C). By comparison, TE was less efficient (best cutoff at $8.2 \mathrm{kPa}$, AUROC $=0.78[0.61-0.91]$ ) (Figure 4D). Ultrasonography and Doppler criteria for SOS were more frequently observed in patients with EBMT criteria but were unable to distinguish between SOS and non-SOS patients (Figure 4E). In order to determine the efficacy of the 2D-SWE measure to improve the predictive value of the current score for SOS diagnosis, we used $3 \times 2$ tables as a diagnostic tool to calculate sensibility, specificity and positive predictive value of 2D-SWE combined with clinical criteria. ${ }^{21,25}$ Classical $2 \times 2$ tables (after exclusion of non-evaluable measures of 2D-SWE) and $3 \times 2$ tables were built to determine the diagnostic value of $2 \mathrm{D}$ SWE measures (Table 3). A worse-scenario approach was calculated, where all non-evaluable measures are classified in the wrong category (absence of SOS diagnosis for patients with SOS and positive SOS diagnosis for patients without SOS). When combined with the EBMT, Baltimore or Seattle scores, a 2D-SWE value higher than $8.1 \mathrm{kPa}$ improved the ability the EBMT score to efficiently diagnose SOS, with a better sensibility (75\%) and specificity $(99 \%)$ for SOS diagnosis (Figure 4F). The addition of 2DSWE improved the positive predictive value of the Seattle score from $19 \%$ to $38 \%$, of the Baltimore score from $33 \%$ to $50 \%$ and of the EBMT score from $40 \%$ to $60 \%$.

\section{Discussion}

After allo-HSCT, liver injury is a major cause of early mortality. $3,4,23,24$ Accurate and early diagnosis is the cornerstone for personalized treatment. This study presents a large real-life cohort of 146 consecutive adult patients who 
A

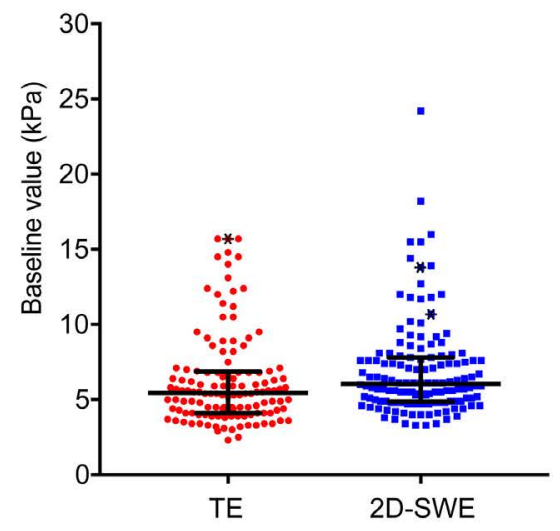

C

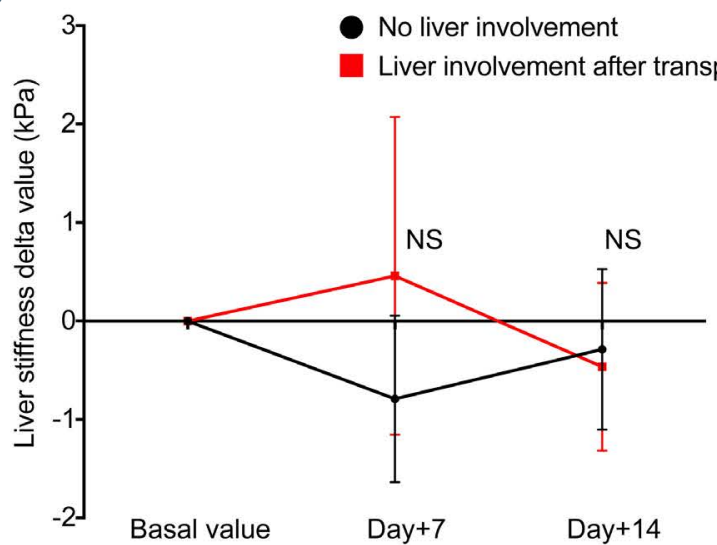

TE
B

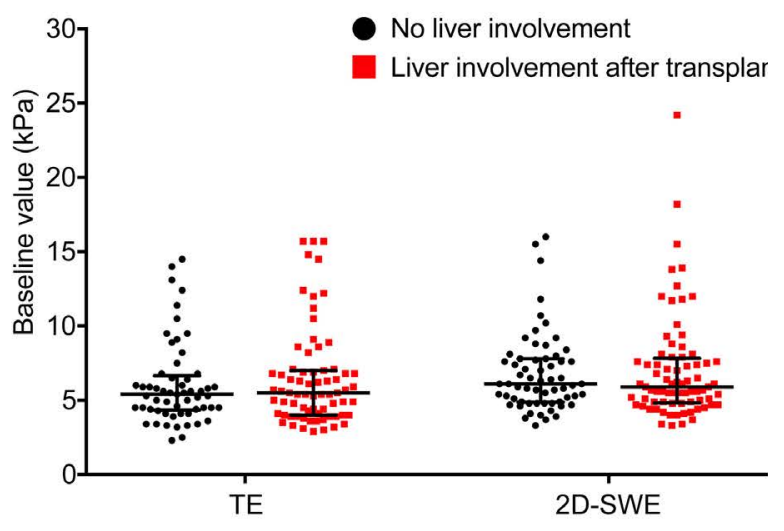

D

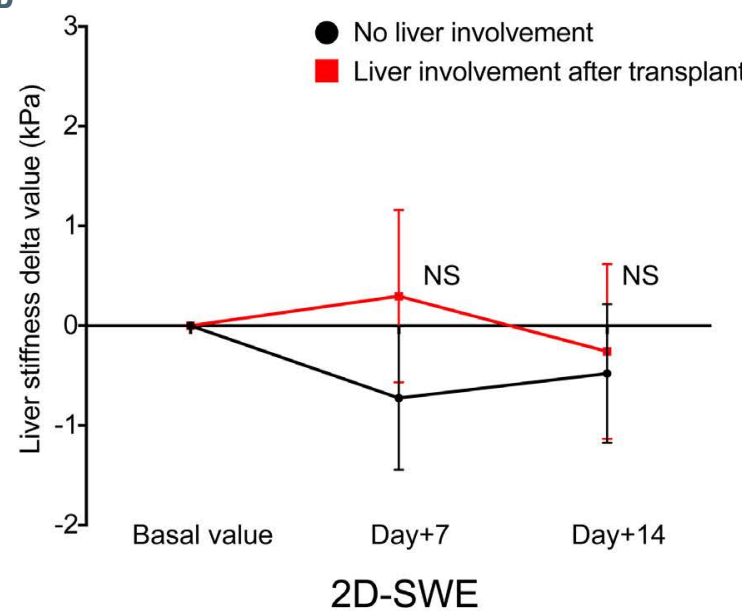

F

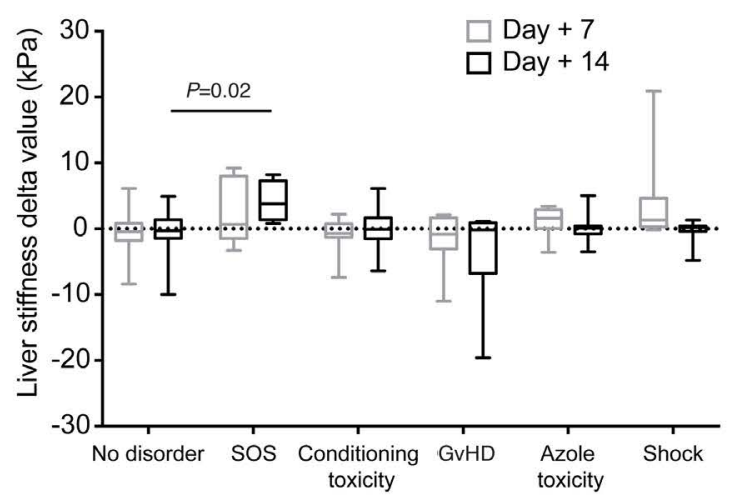

2D-SWE

Figure 3. Baseline and evolution of transient elastography and two-dimensional real-time shear wave elastography after allogeneic hematopoietic stem cell transplantation. (A) Individual baseline measure for transient elastography (TE) (5.4 kPa [range, 4.1-6.8]) and two-dimensional real-time shear wave elastography (2DSWE) $(6.0 \mathrm{kPa}$ [range, 4.9-7.8]) with median and interquartile value. Stars represent a patient with fibrosis before transplantation. (B) No significant difference was observed between baseline value (median and interquartile range) of patients with (red) or without (black) liver involvements (unpaired $t$ test with Holm-Sidak corrections for multiple testing). (C, and D) Sequential evolution of elastography measures compared with baseline with mean and $95 \%$ Confidence Interval (CI). No significant difference was observed between patients with (red line) or without (black line) liver involvements for TE (C) and shear wave (D) at day +7 or at day+14 (unpaired $t$-test with Holm-Sidak correction). For each group, difference between baseline, day +7 and day +14 were not significant (Two-way ANOVA with Dunnet correction for multiple comparison). (E and F) Comparison of elastography delta value (difference between measure and baseline) according to liver diagnosis (box represents median and interquartile values, whiskers are minimum and maximum values) for TE (E) and shear wave (F) (Two-way ANOVA with Dunnet correction multiple comparison). 2D-SWE values were significantly increased in patients with sinusoidal obstruction syndrome (SOS) by comparison with those without liver involvements. 
Table 3. Analyzed results with recalculated $3 \times 2$ tables including non-evaluable measures.

\begin{tabular}{|c|c|c|c|c|c|c|c|c|c|c|}
\hline & \multicolumn{6}{|c|}{$3 \times 2$ table } & \multicolumn{4}{|c|}{ Score performance } \\
\hline & \multicolumn{4}{|c|}{$2 \times 2$ table } & \multicolumn{2}{|c|}{$\begin{array}{l}\text { Non-evaluable results } \\
\text { at patient level for 2D-SWE }\end{array}$} & \multirow[b]{2}{*}{ Se } & \multirow[b]{2}{*}{$S p$} & \multirow[b]{2}{*}{ PPV } & \multirow[b]{2}{*}{ NPV } \\
\hline & $\begin{array}{l}\text { True- } \\
\text { positive }\end{array}$ & $\begin{array}{l}\text { False- } \\
\text { positive }\end{array}$ & $\begin{array}{c}\text { False- } \\
\text { negative }\end{array}$ & $\begin{array}{c}\text { True- } \\
\text { negative }\end{array}$ & $\begin{array}{c}\text { Non- } \\
\text { evaluable } \\
\text { (positive) }\end{array}$ & $\begin{array}{c}\text { Non- } \\
\text { evaluable } \\
\text { (negative) }\end{array}$ & & & & \\
\hline $2 \mathrm{D}-\mathrm{SWE}>8.1 \mathrm{kPa}$ & 4 & 29 & 0 & 97 & 1 & 11 & $80 \%$ & $71 \%$ & $9 \%$ & $99 \%$ \\
\hline EBMT & 6 & 9 & 0 & 131 & NA & NA & $100 \%$ & $94 \%$ & $40 \%$ & $100 \%$ \\
\hline $\mathrm{EBMT}+2 \mathrm{D}-\mathrm{SWE}>8.1 \mathrm{kPa}$ & 3 & 1 & 0 & 135 & 1 & 1 & $75 \%$ & $99 \%$ & $60 \%$ & $99 \%$ \\
\hline Baltimore & 4 & 8 & 2 & 132 & NA & NA & $67 \%$ & $94 \%$ & $33 \%$ & $99 \%$ \\
\hline Baltimore + 2D-SWE $>8.1 \mathrm{kPa}$ & 3 & 2 & 2 & 132 & 1 & 1 & $50 \%$ & $98 \%$ & $50 \%$ & $98 \%$ \\
\hline Seattle & 5 & 22 & 1 & 118 & NA & NA & $83 \%$ & $84 \%$ & $19 \%$ & $99 \%$ \\
\hline Seattle + 2D-SWE >8.1 kPa & 3 & 3 & 1 & 131 & 1 & 2 & $60 \%$ & $96 \%$ & $38 \%$ & $99 \%$ \\
\hline
\end{tabular}

2D-SWE: two-dimensional real-time shear wave elastography; EBMT: European Bone Marrow Transplantation; NA: not applicable; Se: sensibility; Sp: specificity; PPV: positive predictive value; NPV: negative predictive value.

underwent allo-HSCT. In our study, elastography was significantly and specifically increased in patients who developed a SOS. In association with standard SOS scores, 2DSWE improved the specificity of these scores and their positive predictive value (thus decreasing the risk of over treating patients with expensive drugs with potential sideeffects).

In this patient cohort, liver biological abnormalities were frequent after transplantation. Around 39\% of patients developed a hyperbilirubinemia, an incidence similar to what has previously been described after alloHSCT ${ }^{3,4}$ Hyperbilirubinemia has been shown to be predictive of $\mathrm{TRM}^{3}$ and liver injury is associated with a high mortality rate after transplantation. ${ }^{26} \mathrm{We}$ previously reported that clinical and biological features are poorly predictive of liver lesions and that liver biopsy is a useful approach to improve diagnosis of liver involvements in selected patients. ${ }^{5}$ Improving accuracy of liver involvements after allo-HSCT is challenging and we currently lack reproducible and specific markers easily available for all patients. Elastography has been widely developed as a non-invasive and quantitative tool for liver involvements, especially fibrosis and cirrhosis. ${ }^{27}$ The baseline values measured in this study were quite similar to those of healthy subjects previously published for TE (5.49+/-1.59 $\mathrm{kPa})^{28}$ or $2 \mathrm{D}-\mathrm{SWE}(5.19+/-1.03 \mathrm{kPa}){ }^{29}$ However, a significant proportion of patients had higher basal value before transplantation, especially in those with pre-existing liver involvements. In a smaller mixed cohort of patients who underwent autologous $(n=37)$ or allo-HSCT $(n=30)$, baseline transient elastography values could predict the occurrence of hyperbilirubinemia after transplantation. ${ }^{30}$ In addition, it has been shown that transient elastography and point shear wave values before allo-HSCT were higher in patients developing severe or life-threatening liver complications after allo-HSCT. ${ }^{31}$ Our study did not identify baseline values as a marker for subsequent liver injury after allo-HSCT, but baseline measures might help the interpretation of post-transplantation measures.

This study was initially designed to explore elastography for the diagnosis of all early liver complications occurring during the first 100 days after transplantation. In our study, the liver stiffness measure was not significantly increased in patients with GvHD, DILI or infectious disease. SOS was the only complication in which $2 \mathrm{D}-\mathrm{SWE}$ measures were significantly increased as compared to baseline values. Only six cases of SOS were confirmed in this cohort, a low incidence that limits the interpretation of our data on 2D-SWE measures for early diagnosis of SOS. In rat models of SOS, point shear wave velocity with ARFI was increased in animals with SOS as compared to controls and was correlated with a high SOS histological score, inflammation and congestion, but not with fibrosis. ${ }^{32}$ Inflammation or congestion due to viral hepatitis,${ }^{33}$ biliary obstruction, ${ }^{34}$ cardiac failure, ${ }^{35}$ and acute lymphoid leukemia relaps ${ }^{36}$ have been reported to be associated with elevated TE. The TE value mainly depends on tissue stiffness with a region of interest (ROI) of 40-50 $\mathrm{mm}^{2}$. By contrast, 2D-SWE measure depends on tissue stiffness and viscosity with ROI of $100 \mathrm{~mm}^{2}$ or more. ${ }^{16}$ As SOS is a heterogeneous vascular liver disease characterized by progressive sinusoid vessel obstruction that could affect viscosity, it could explain why 2D-SWE was more efficient than TE to diagnose SOS. The incidence of an unreliable TE measure (>10\%) could decrease the diagnosis performance in comparison to 2D-SWE. Finally, 2DSWE was not operator-dependent in our study as in others. ${ }^{37}$

Many techniques of elastography have been proposed to predict hepatic complications ${ }^{30,31}$ or to diagnose SOS after allo-HSCT. ${ }^{36,38,39}$ In a pediatric cohort of 22 patients, sequential measures of TE after allo-HSCT showed increased TE measures 3 to 6 days before clinical signs in five SOS cases (based on Seattle or Baltimore classification). ${ }^{38}$ In a second pediatric series of 25 patients, sequential 2D-SWE velocity at day +5 and day+14 in five SOS patients was higher than in the control population. ${ }^{39}$ In the adult setting, one single center study including 78 patients after allo-HSCT evaluated the usefulness of sequential measures of TE for SOS diagnoses. ${ }^{36} \mathrm{TE}$ was significantly increased in four patients with SOS.

Currently, SOS diagnosis relies on clinical criteria that have low specificity. To our knowledge, there is no published evaluation of the sensibility, specificity or predictive value of EBMT, Seattle or Baltimore criteria, using liver biopsy as a gold standard method for SOS diagnosis. ${ }^{8}$ In our cohort, one third of patients with EBMT criteria had a liver biopsy and the systematic retrospective review of their medical history helped us to better estimate the predictive value of each clinical score. ${ }^{40}$ For patients who did 
A

Number of patients with SOS criteria

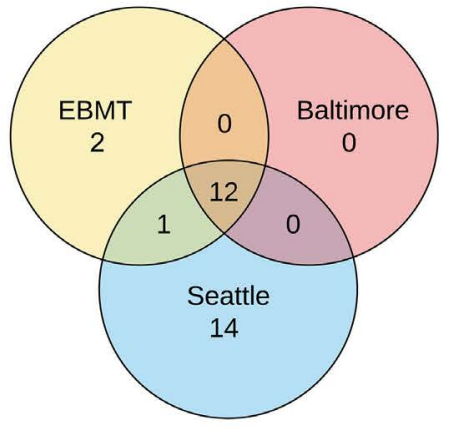

C

2D-Shear wave Day+14
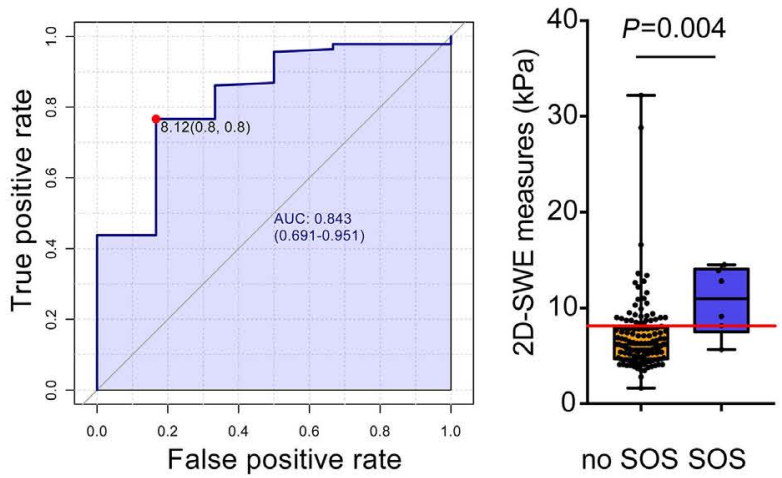

E

Day +7
B

Number of patients with final SOS diagnosis

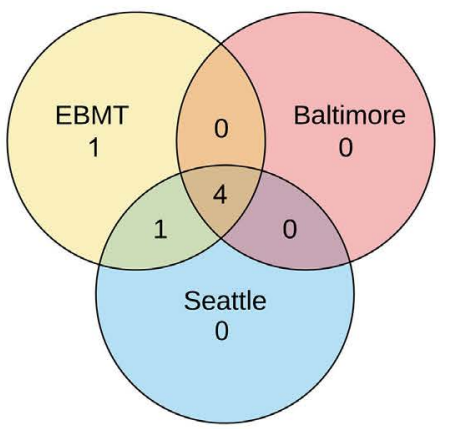

D
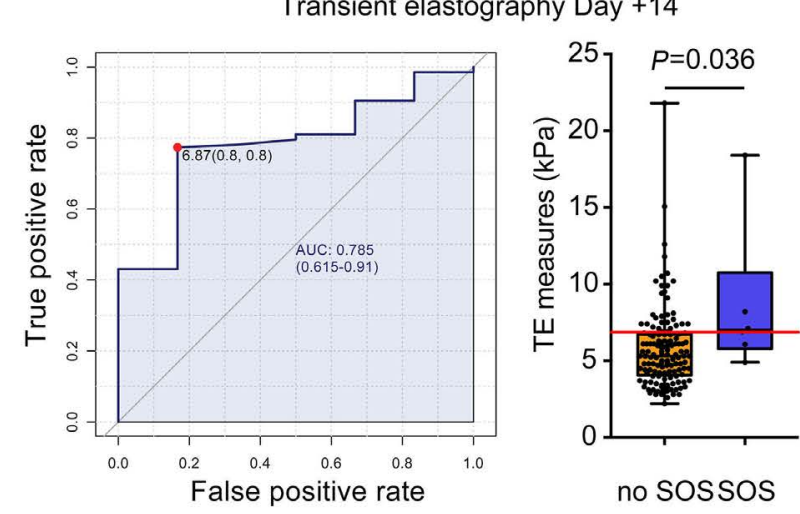

no sossos

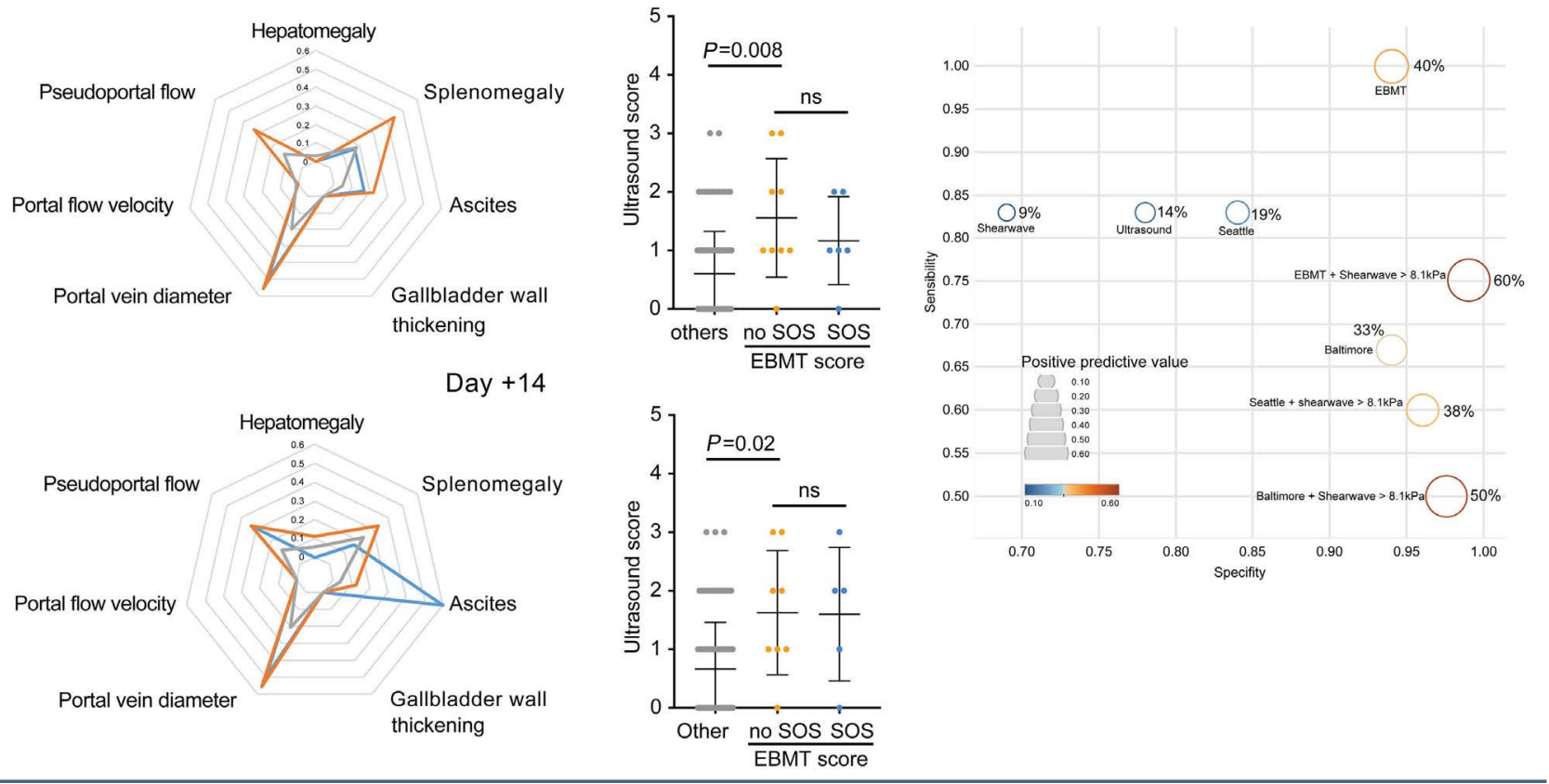

Figure 4. Two-dimensional real-time shear wave elastography measures improve sinusoidal obstruction syndrome diagnosis. (A and B) Venn diagram representing the distribution of patients with European Society for Blood and Marrow Transplantation (EBMT), Seattle and/or Baltimore Criteria in this cohort (A) and those with final diagnosis of sinusoidal obstruction syndrome (SOS) in each group (B). (C and D) ROC curves for day+14 two-dimensional real-time shear wave elastography (2DSWE) and transient elastography (TE) measures, and box plot of individual values (box represents median and interquartile values, whiskers are minimum and maximum values) in patients with (blue) or without SOS (yellow) for shear wave (A) and TE (B) compared with Mann-Whitney U test. (E) Radar plot of individual ultrasound criteria and box plot of ultrasonography scores (calculated by adding one point per criteria) for patients with no EBMT criteria (gray lines) and with EBMT criteria without (orange lines) or with SOS diagnosis (blue lines) compared with Kruskal Wallis test and Dunn's correction for multiple testing. (F) Representation of sensibility, specificity, and positive predictive rate (PPR) (color circle, large and red circle are associated with greatest PPR). Addition of 2D-SWE measure with a cutoff value at $8.1 \mathrm{kPa}$ improved the PPR value of the Seattle score from $19 \%$ to $38 \%$, of the Baltimore score from $33 \%$ to $50 \%$ and of the EMBT score from $40 \%$ to $60 \%$. 
not have a biopsy, the final diagnosis relied on a retrospective analysis of all medical data, of clinical evolution with treatments, and on consensual diagnosis criteria if available. However, the lack of gold standard criteria for most of diagnoses, such as GvHD, SOS or DILI, could bias conclusions as there is currently no reliable tool to avoid misdiagnoses. An unreliable TE or 2D-SWE value is classically estimated to be included between $5 \%$ and $10 \%$, a range very similar to what was observed in our cohort. ${ }^{41}$ Using an intention to diagnose approach helped us to estimate the suitability of the liver stiffness measurement in addition to clinical criteria for SOS diagnosis. Our results confirm the interest of liver stiffness measures and strongly suggest that 2D-SWE significantly improved the positive predictive value of clinical scores for SOS diagnosis after allo-HSCT. Increasing the positive predictive value of current criteria for SOS is critical to avoid useless and potentially toxic treatment in patient with comorbidities.

Thus, 2D-SWE appears as a promising, non-invasive, quantitative, safe, and reproducible technique allowing an early and accurate diagnosis of SOS. 2D-SWE measures combined with classical scores such as Baltimore or EBMT criteria could significantly help discriminating SOS from other post-transplantation early liver injury. Prospective multi-center trials would be necessary to confirm these results and to broadly evaluate the impact of liver stiffness measures on management and treatment of liver involvements after allo-HSCT.

\section{Disclosures}

No conflicts of interest to disclose.

\section{Contributions}

PED and DM collected, analyzed data and performed statistics analysis; $P B, A M Z$ and $M D B$ performed ultrasonography, Doppler and elastrography measurements; PER, SP, ASG, MR, $R P L, A P, F S F, A X, P H P$ and $D V$ provided data and commented manuscript; PED, GS and DM wrote the manuscript; GS and $D M$ conceived the project and supervised the work; all authors approved the manuscript.

\section{References}

1. Gooley TA, Chien JW, Pergam SA, et al. Reduced mortality after allogeneic hematopoietic-cell transplantation. N Engl J Med 2010;363(22):2091-2101.

2. McDonald GB. Hepatobiliary complications of hematopoietic cell transplantation, 40 years On. Hepatology 2010;51(4):14501460.

3. Gooley TA, Rajvanshi P, Schoch HG, McDonald GB. Serum bilirubin levels and mortality after myeloablative allogeneic hematopoietic cell transplantation. Hepatology 2005;41(2):345-352.

4. Hogan WJ. Hepatic injury after nonmyeloablative conditioning followed by allogeneic hematopoietic cell transplantation: a study of 193 patients. Blood 2004; 103(1):78-84.

5. Ruggiu M, Bedossa P, Rautou PE, et al. Utility and safety of liver biopsy in patients with undetermined liver blood test anomalies after allogeneic hematopoietic stem cell transplantation: a monocentric retrospective cohort study. Biol Blood Marrow Transplant 2018:24(12):2523-2531

6. Lassau $\mathrm{N}$, Leclère J, Auperin $\mathrm{A}$, et al. Hepatic veno-occlusive disease after myeloablative treatment and bone marrow transplantation: value of gray-scale and Doppler US in 100 patients. Radiology 1997;204(2):545-552.

7. Nishida M, Kahata K, Hayase E, et al. Novel Ultrasonographic scoring system of sinusoidal obstruction syndrome after hematopoietic stem cell transplantation. Biol Blood Marrow Transplant J Am Soc Blood Marrow Transplant 2018; 24(9):1896-1900

8. EASL Clinical Practice Guidelines: vascular diseases of the liver. J Hepatol 2016; 64(1):179-202

9. Teefey SA, Brink JA, Borson RA, Middleton WD. Diagnosis of venoocclusive disease of the liver after bone marrow transplantation: value of duplex sonography. AJR Am J Roentgenol 1995;164(6):1397-1401.

10. McCarville MB, Hoffer FA, Howard SC, Goloubeva O, Kauffman WM. Hepatic veno-occlusive disease in children undergoing bone-marrow transplantation: usefulness of sonographic findings. Pediatr Radiol
2001;31(2):102-105.

11. Shiina T, Nightingale KR, Palmeri ML, et al. WFUMB guidelines and recommendations for clinical use of ultrasound elastography: part 1: basic principles and terminology. Ultrasound Med Biol 2015;41(5):11261147 .

12. European Association for Study of Liver, Asociacion Latinoamericana para el Estudio del Higado. EASL-ALEH Clinical Practice Guidelines: non-invasive tests for evaluation of liver disease severity and prognosis. J Hepatol 2015;63(1):237-264.

13. Dietrich CF, Trenker C, Fontanilla T, et al. New ultrasound techniques challenge the diagnosis of sinusoidal obstruction syndrome. Ultrasound Med Biol 2018; 44(11):2171-2182.

14. Bacigalupo A, Ballen K, Rizzo D, et al. Defining the intensity of conditioning regimens: working definitions. Biol Blood Marrow Transplant J Am Soc Blood Marrow Transplant 2009;15(12):1628-1633.

15. Armand P, Gibson CJ, Cutler C, et al. A disease risk index for patients undergoing allogeneic stem cell transplantation. Blood 2012;120(4):905-913

16. Dietrich CF, Bamber J, Berzigotti A, et al. EFSUMB guidelines and recommendations on the clinical use of liver ultrasound elastography, update 2017 (long version). Ultraschall Med Stuttg Ger 1980 2017; 38(4):e16-e47.

17. Glucksberg H, Storb R, Fefer A, et al. Clinical manifestations of graft-versus-host disease in human recipients of marrow from HL-A-matched sibling donors. Transplantation 1974;18(4):295-304.

18. McDonald GB, Hinds MS, Fisher LD, et al. Veno-occlusive disease of the liver and multiorgan failure after bone marrow transplantation: a cohort study of 355 patients. Ann Intern Med 1993;118(4):255-267.

19. Jones RJ, Lee KS, Beschorner WE, et al. Venoocclusive disease of the liver following bone marrow transplantation. Transplantation 1987;44(6):778-783.

20. Mohty M, Malard F, Abecassis M, et al. Revised diagnosis and severity criteria for sinusoidal obstruction syndrome/venoocclusive disease in adult patients: a new classification from the European Society for Blood and Marrow Transplantation. Bone
Marrow Transplant 2016;51(7):906-912.

21. Cohen JF, Korevaar DA, Altman DG, et al. STARD 2015 guidelines for reporting diagnostic accuracy studies: explanation and elaboration. BMJ Open 2016; 6(11): e012799.

22. Andrade RJ, Aithal GP, Björnsson ES, et al. EASL clinical practice guidelines: druginduced liver injury. J Hepatol 2019; 70(6):1222-1261.

23. Schuetz GM, Schlattmann P, Dewey M. Use of $3 \times 2$ tables with an intention to diagnose approach to assess clinical performance of diagnostic tests: meta-analytical evaluation of coronary CT angiography studies. BMJ 2012;345e6717.

24. McDonald GB, Sharma P, Matthews DE, Shulman HM, Thomas ED. Venocclusive disease of the liver after bone marrow transplantation: diagnosis, incidence, and predisposing factors. Hepatol Baltim Md 1984;4(1):116-122.

25. Schuetz GM, Schlattmann P, Dewey M. Use of $3 \times 2$ tables with an intention to diagnose approach to assess clinical performance of diagnostic tests: meta-analytical evaluation of coronary CT angiography studies. BMJ;345.

26. Sakai M, Strasser SI, Shulman HM McDonald SJ, Schoch HG, McDonald GB. Severe hepatocellular injury after hematopoietic cell transplant: incidence, etiology, and outcome. Bone Marrow Transplant 2009;44(7):441-447.

27. Dighe M, Bruce M. Elastography of diffuse liver diseases. Semin Roentgenol 2016, 51(4):358-366.

28. Roulot D, Czernichow S, Le Clésiau $\mathrm{H}$, Costes J-L, Vergnaud A-C, Beaugrand M. Liver stiffness values in apparently healthy subjects: influence of gender and metabolic syndrome. J Hepatol 2008:48(4):606-613.

29. Petzold G, Hofer J, Ellenrieder V, Neesse A, Kunsch S. Liver stiffness measured by 2 dimensional shear wave elastography: prospective evaluation of healthy volunteers and patients with liver cirrhosis. Ultrasound Med Off J Am Inst Ultrasound Med 2019;38(7):1769-1777.

30. Auberger J, Graziadei I, Clausen J, Vogel W, Nachbaur D. Non-invasive transient elastography for the prediction of liver toxicity following hematopoietic SCT. Bone 
Marrow Transplant 2013;48(1):159-160.

31. Karlas T, Weiße T, Petroff D, et al. Predicting hepatic complications of allogeneic hematopoietic stem cell transplantation using liver stiffness measurement. Bone Marrow Transplant 2019;1-9.

32. Park SH, Lee SS, Sung J-Y, et al. Noninvasive assessment of hepatic sinusoidal obstructive syndrome using acoustic radiation force impulse elastography imaging: a proof-of-concept study in rat models. Eur Radiol 2018;28(5):2096-2106.

33. Arena U, Vizzutti F, Corti G, et al. Acute viral hepatitis increases liver stiffness values measured by transient elastography. Hepatology 2007;47(2):380-384.

34. Millonig G, Reimann FM, Friedrich S, et al. Extrahepatic cholestasis increases liver stiffness (FibroScan) irrespective of fibrosis. Hepatology 2008;48(5):1718-1723.

35. Colli A, Pozzoni P, Berzuini A, et al.
Decompensated chronic heart failure: increased liver stiffness measured by means of transient elastography. Radiology 2010;257(3):872-878.

36. Colecchia A, Ravaioli F, Sessa M, et al. Liver stiffness measurement allows early diagnosis of veno-occlusive disease/sinusoidal obstruction syndrome in adult patients who undergo hematopoietic stem cell transplantation: results from a monocentric prospective study. Biol Blood Marrow Transplant 2019;25(5):995-1003.

37. Poynard T, Pham T, Perazzo H, et al. Realtime shear wave versus transient elastography for predicting fibrosis: applicability, and impact of inflammation and steatosis. a non-invasive comparison. PLOS ONE 2016;11(10):e0163276.

38. Colecchia A, Marasco G, Ravaioli F, et al. Usefulness of liver stiffness measurement in predicting hepatic veno-occlusive dis- ease development in patients who undergo HSCT. Bone Marrow Transplant 2017;52 (3):494-497.

39. Reddivalla N, Robinson AL, Reid KJ, et al Using liver elastography to diagnose sinusoidal obstruction syndrome in pediatric patients undergoing hematopoetic stem cell transplant. Bone Marrow Transplant 2018;1-8.

40. Volin L, Niittyvuopio R, Heiskanen J, et al. Diagnosis of veno-occlusive disease/sinusoidal obstruction syndrome of the liver: problems of interpretation. Bone Marrow Transplant 2016;51(12):1633-1635.

41. Kim DW, Suh CH, Kim KW, Pyo J, Park C, Jung SC. Technical performance of twodimensional shear wave elastography for measuring liver stiffness: a systematic review and meta-analysis. Korean J Radiol 2019;20(6):880-893. 